\title{
Bourses SFRP
}

La SFRP a attribué une bourse à ces jeunes étudiants pour leur stage de mastère $2^{e}$ année de recherche. Nous avons le plaisir de publier dans cette rubrique destinée aux jeunes chercheurs un résumé de leur stage. Nous leur souhaitons de continuer brillamment leur carrière en souhaitant qu'ils restent attachés à la radioprotection.

Évaluation des doses dues aux neutrons secondaires reçues par les patients traités par protonthérapie.

Cette étude a été réalisée dans le cadre de mon Master 2, à l'Institut de radioprotection et de sûreté nucléaire (IRSN) au Laboratoire de dosimétrie des rayonnements ionisants, et a bénéficié du soutien de la Société française de radioprotection (SFRP).

La protonthérapie est une technique spécifique de radiothérapie, utilisant un faisceau de protons en raison de leurs propriétés balistiques. Lors d'un traitement par protonthérapie, le faisceau de protons subit des interactions nucléaires avec la matière, créant principalement des neutrons. Cette étude a pour but d'évaluer les doses dues à ce rayonnement neutronique secondaire reçues par le patient, dans le cas de traitements pédiatriques intracrâniens en conditions cliniques. Elle se déroule en trois étapes : celle de modélisation de la ligne, celle de validation de la modélisation grâce à des comparaisons entre calculs et mesures, et enfin l'étape d'étude de la dose déposée dans des fantômes pédiatriques voxelisés. Une étude précédente a déjà été menée au Centre de protonthérapie de l'institut Curie (ICPO) dans la salle de traitements ophtalmologiques sur des adultes à une énergie d'environ $78 \mathrm{MeV}$. L'étude actuelle est réalisée dans une autre salle de l'ICPO équipée d'un bras isocentrique, pour des traitements pédiatriques intracrâniens d'une énergie d'environ $178 \mathrm{MeV}$.

La protonthérapie présente deux intérêts majeurs: les protons diffusent très peu latéralement, et déposent la dose de façon localisée selon un pic (pic de Bragg) en fin de parcours, créant une pénombre distale quasi nulle. Pour cela, elle est indiquée pour les tumeurs de situation anatomique particulière, avec des tissus sains avoisinants indispensables à protéger. Il existe deux techniques différentes pour conformer le faisceau à la zone tumorale : la diffusion passive, qui consiste à insérer sur la trajectoire des protons différents composants qui le façonnent, technique qui est utilisée à l'ICPO, et la diffusion active qui fait appel au balayage d'un fin faisceau de protons en position, en intensité et en énergie pour traiter toute la zone tumorale.

La modélisation de la salle de traitement et du faisceau est réalisée avec le logiciel de simulation Monte-Carlo MCNPX. C'est une technique mathématique permettant de calculer une valeur numérique en utilisant des procédés aléatoires, pour un problème n'ayant pas de solution analytique. Elle permet le transport de trente-quatre types de particules (ions et particules électromagnétiques inclus) dont les interactions avec la matière sont aléatoires, sur 
une gamme d'énergie d'environ $1 \mathrm{keV}$ à $100 \mathrm{GeV}$, dépendant de sections efficaces disponibles sous la forme de base de données et basées sur des lois de probabilité.

Le modèle est validé expérimentalement à l'aide de plusieurs mesures effectuées en conditions cliniques. Tout d'abord, on vérifie la correspondance du rendement en profondeur calculée par MCNPX (dont les incertitudes sont au maximum de $2 \%$ ) et mesurée à l'ICPO avec les chambres d'ionisation adaptées (dont les incertitudes sont d'environ $3 \%$ ) : elle est conforme sur le plateau de dose, et diffère jusqu'à un facteur 5 dans la pénombre distale. Ensuite, on compare les profils latéraux : de même que précédemment, les calculs et les mesures sont conformes sur le plateau de dose, et diffèrent dans les pénombres latérales, le plus souvent d'un facteur 1,5 à 2,5. Enfin, on utilise un fantôme physique anthropomorphe RANDO $^{\circledR}$, dans lequel on place des CR-39 afin de mesurer la dose déposée par les neutrons. Cette vérification est plus délicate que la précédente. En effet, les doses secondaires dues aux neutrons dépendent de paramètres plus difficiles à déterminer que les doses du faisceau incident : les calculs avec MCNPX mettent du temps à converger, et les CR-39 nécessitent un étalonnage fortement dépendant de l'énergie qui introduit des incertitudes supplémentaires. Finalement, un écart limité à un facteur 2,5 entre mesures et calculs est observé, pour des doses qui sont de l'ordre du milligray (environ $2 \mathrm{mGy}$ dans la thyroïde, et 0,5 dans les ovaires).

L'accord entre les résultats du modèle et les mesures est considéré acceptable en regard des objectifs de l'étude : il est excellent pour les distributions de dose due aux protons dont les calculs reproduisent très bien l'allure du faisceau, et l'écart entre calculs et mesures des doses dues aux neutrons dans le fantôme $\mathrm{RANDO}^{\circledR}$ (résultats préliminaires) est correct vis-à-vis des estimations de radioprotection, le plus souvent majorantes. On valide ainsi la modélisation de la ligne de traitement, et les écarts entre calculs et mesures ont pu être quantifiés.

Le modèle est ensuite exploité pour réaliser des calculs de doses dues aux neutrons secondaires dans un fantôme pédiatrique voxelisé de référence. La validation de la modélisation permet la simulation de traitements réalistes. Les fantômes sont utilisés pour calculer des doses dans l'ensemble des organes hors du champ de traitement de patients de différents âges : cette étude est en cours d'approfondissement. À terme, il sera possible de comparer différentes stratégies de traitement par protonthérapie entre elles, différentes installations entre elles, d'améliorer certaines protections radiologiques, mais aussi d'évaluer les risques post traitement.

Alix AUBÉ 


\section{Utilisation de codes de simulation Monte-Carlo (MCNPX, PENELOPE) dans le cadre de la radioprotection en médecine nucléaire.}

Cette étude a été réalisé au Centre hospitalier Princesse Grace à Monaco.

La radioprotection est au cœur des services de médecine nucléaire de par la large gamme d'exposition en termes d'énergie et de particule. La démocratisation du TEP et l'introduction de nouveaux traceurs (fluorodopa, fluorocholine, fluorure de sodium) augmentent l'utilisation du fluor 18 et donc l'exposition du personnel médical et paramédical au rayonnement gamma de $511 \mathrm{keV}$.

Nous nous proposons d'étudier l'efficacité du matériel de radioprotection tel que les tabliers plombés (de $0,5,0,35$, et $0,25 \mathrm{~mm}$ de plomb) pour les sources d'émission gamma les plus utilisées $\left({ }^{99 \mathrm{~m}} \mathrm{Tc},{ }^{123} \mathrm{I},{ }^{131} \mathrm{I},{ }^{111} \mathrm{In},{ }^{67} \mathrm{Ga},{ }^{201} \mathrm{Tl}\right)$. De plus, il serait intéressant d'étudier l'utilité du port d'un tablier de $0,5 \mathrm{~mm}$ de plomb pour une source de ${ }^{18} \mathrm{~F}$ et de mettre en évidence les causes d'un éventuel surplus de dose absorbée à la peau.

Pour cela, nous avons employé des codes de simulation Monte-Carlo (MCNPX 2.4.0, PENELOPE 2008) qui permettent, après une validation expérimentale, d'obtenir des informations physiques et dosimétriques précises. Cela nous permet ainsi de mettre en évidence les phénomènes physiques induits par le plomb sur les particules sources. Pour juger de l'efficacité des tabliers, nous nous sommes basés sur les valeurs de Hp10 et Hp0,07 représentant respectivement la dose absorbée en profondeur $(a ̀ ~ 1 \mathrm{~cm}$ ) et la dose absorbée à la peau (à $70 \mu \mathrm{m})$. Nous avons modélisé dans un premier temps le patient par une cuve à eau puis par un fantôme anthropomorphique dont les structures et la composition des organes se rapprochent d'avantage de la réalité.

Nos résultats, aux travers de rendements en profondeur et de spectres, ont montré que pour le ${ }^{18} \mathrm{~F}$, un tablier de $0,5 \mathrm{~mm}$ de plomb permet de réduire la dose absorbée en profondeur (Hp10) de $9 \%$ mais augmente la dose absorbée à la peau $(\mathrm{Hp} 0,07)$ de $87 \%$. Par contre, pour une même épaisseur de tablier mais avec une exposition au ${ }^{99 \mathrm{~m}} \mathrm{Tc}$, la dose absorbée en profondeur est réduite de $74 \%$ alors que la dose absorbée à la peau n'est augmentée que de 1,6\%, ce qui rend le tablier efficace. Le tableau suivant rassemble les valeurs de dose absorbée en profondeur pour les autres radionucléides en fonction des trois épaisseurs de plomb.

Nous avons mis en évidence la cause du surplus de dose absorbée à la peau grâce à la décomposition du rendement en profondeur en fonction de l'origine des particules. La figure 1 représente l'énergie déposée dans la cuve à eau en fonction de l'origine des particules.

Nous pouvons voir que la dose absorbée en profondeur est uniquement due aux photons primaires. En revanche l'énergie déposée à la peau est due à la fois aux photons primaires et aux électrons secondaires. Le surplus de dose absorbée à la peau est donc uniquement dû aux électrons créés dans le tablier par effet photoélectrique ou Compton. Le rendement somme (photons primaires + électrons secondaires) et le rendement du fluor 18 après $0,5 \mathrm{~mm}$ de plomb sont en concordance quasi-parfaite ce qui montre bien que l'énergie déposée par les photons diffusés (Compton et $\mathrm{X}$ caractéristiques) est négligeable. 
TABLEAU I

Atténuation de dose absorbée en profondeur pour les autres radionucléides en fonction des différentes épaisseurs de plomb.

\begin{tabular}{|c|c|c|}
\hline Isotope & Épaisseur de plomb (mm) & Atténuation Hp10 (\%) \\
\hline \multirow{3}{*}{${ }^{99 \mathrm{~m}} \mathrm{Tc}$} & 0,5 & $-73,1$ \\
\hline & 0,35 & $-60,0$ \\
\hline & 0,25 & $-47,2$ \\
\hline \multirow{3}{*}{${ }^{111}$ In } & 0,5 & $-38,1$ \\
\hline & 0,35 & $-29,0$ \\
\hline & 0,25 & $-21,9$ \\
\hline \multirow{3}{*}{${ }^{123} \mathrm{I}$} & 0,5 & $-61,6$ \\
\hline & 0,35 & $-52,1$ \\
\hline & 0,25 & $-44,2$ \\
\hline \multirow{3}{*}{${ }^{67} \mathrm{Ga}$} & 0,5 & $-40,9$ \\
\hline & 0,35 & $-33,8$ \\
\hline & 0,25 & $-27,3$ \\
\hline \multirow{3}{*}{${ }^{131} \mathrm{I}$} & 0,5 & $-12,2$ \\
\hline & 0,35 & $-8,8$ \\
\hline & 0,25 & $-6,5$ \\
\hline \multirow{3}{*}{${ }^{201} \mathrm{Tl}$} & 0,5 & $-74,7$ \\
\hline & 0,35 & $-62,9$ \\
\hline & 0,25 & $-51,6$ \\
\hline
\end{tabular}

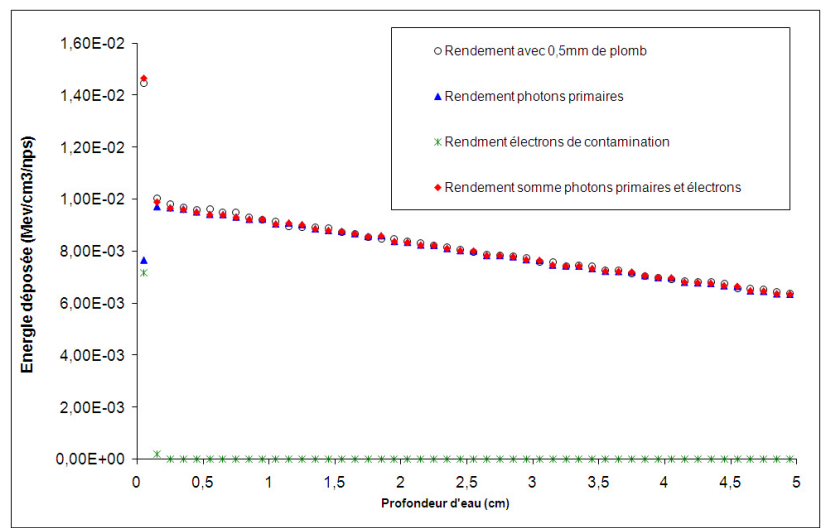

Figure 1 - Décomposition du rendement en profondeur d'une source de ${ }^{18} \mathrm{~F}$ après $0,5 \mathrm{~mm}$ de plomb.

Finalement, grâce aux résultats de la mise en place de codes Monte-Carlo, nous avons pu, aux travers des phénomènes physiques, expliquer la nécessité ou non du port de matériel de radioprotection en fonction des radioéléments utilisés.

Nicolas GARNIER 